\title{
El rol de la responsabilidad social empresarial en el modelo de inclusión laboral para víctimas y desmovilizados del conflicto armado colombiano
}

\section{Sara Sierra*}

Fecha de recibido: 22 de julio de 2020 Fecha de aprobado: 16 de octubre de 2020

Para citar este artículo: Sierra, S. (2021). El rol de la responsabilidad social empresarial en el modelo de inclusión laboral para víctimas y desmovilizados del conflicto armado colombiano. Revista Universidad \& Empresa, 23(40), 1-23. https://doi.org/10.12804/ revistas.urosario.edu.co/empresa/a.9392

\section{Resumen}

Varios estudios han analizado los efectos desfavorables que deja el conflicto armado en Colombia y sus repercusiones en los ámbitos sociales y empresariales En la actualidad, el Gobierno ha implementado, junto con el sector empresarial, un modelo de inclusión laboral para las víctimas del conflicto armado y desmovilizados, a fin de permitirles su reincorporación a la vida civil por medio de un empleo digno. El artículo hace una revisión sistemática de la literatura dedicada a la conceptualización de la inclusión laboral. Ello permitió identificar como objetivos de investigación elementos que forman parte de las prácticas empresariales que se llevan a cabo para la construcción de paz, la vinculación laboral de víctimas y desmovilizados, así como la responsabilidad social empresarial (RSE). Los resultados obtenidos, si bien se describen algunos casos donde la RSE tiene apoyo de algunos empresarios en el desarrollo de programas de inclusión laboral

Economista. MBA de la Universidad del Rosario (Colombia). Máster en Liderazgo Internacional de la Universidad EADA (España). Joven investigadora, Escuela de Administración y Negocios, Universidad del Rosario. Docente universitaria. Correo electrónico: sara.sierra@urosario.edu.co 
El rol de la responsabilidad social empresarial en el modelo de inclusión laboral para víctimas y desmovilizados del conflicto armado colombiano

para las víctimas y desmovilizados del conflicto, evidenciaron falta oportunidades y de qué manera se pueden crear partiendo de iniciativas de cambio y construcción de paz.

Palabras clave: inclusión laboral; responsabilidad social corporativa; reintegración; víctimas del conflicto.

\title{
The Role of Corporate Social Responsibility in the Model of Labor Inclusion for Victims and Demobilized People of the Colombian Armed Conflict
}

\begin{abstract}
Studies have analyzed the unfavorable effects of the armed conflict in Colombia and the repercussions in the social and business spheres. Currently, the government has implemented, together with the business sector, a model of labor inclusion for victims of the armed conflict and demobilized, to allow them to return to civilian life through decent employment. A systematic literature review dedicated to the conceptualization of labor inclusion was done, which allowed identifying as research objectives elements that are part of the business practices carried out for the construction of peace, labor relations of victims with demobilized persons, as well as Corporate Social Responsibility (CSR). Although there is support from some businesspeople who have labor inclusion programs for victims and demobilized people of the conflict, the opportunities should be more. This work concludes suggesting characteristics that highlight the factors applicable to different organizations.
\end{abstract}

Keywords: Labour inclusion; business model; reintegration; victims of the armed conflict.

\section{O papel da responsabilidade social empresarial no modelo de inclusão laboral para vítimas e desmobilizados do conflito armado colombiano}

\section{Resumo}

Estudos têm analisado os efeitos desfavoráveis deixados pelo conflito armado na Colômbia e as repercussões no âmbito social e empresarial. Atualmente o governo implementou, juntamente com o setor empresarial, um modelo de inclusão laboral para as vítimas do conflito armado e desmobilizados, com o intuito de permitir sua reintegração à vida civil por meio de um emprego digno. Se faz uma revisão sistemática de literatura dedicada à conceitualização da inclusão laboral. Permitindo identificar como objetivos da pesquisa, elementos que fazem parte das práticas empresariais que são adotadas para a construção da paz, vinculação laboral de vítimas e desmovilizados, como também da responsabilidade social empresarial (RSE). Os resultados obtidos apontam que, embora sejam descritos alguns casos, em que a RSE tem apoio de alguns empresários no desenvolvimento de programas de inclusão laboral para as vítimas e desmobilizados do conflito, também se apresenta a falta de oportunidades e como estas poderiam ser criadas, partindo-se de iniciativas de mudança e construção da paz.

Palavras-chave: inclusão laboral; responsabilidade social corporativa; reintegração; vítimas do conflito. 


\section{Introducción}

En las últimas décadas (1948-2009) y a lo largo de su historia, veinticinco países han sobrellevado un conflicto armado interno (como lo es el caso colombiano) y han recurrido a mecanismos de respaldo de diferentes organizaciones internacionales, apoyo de países amigos, así como a empresarios encargados de dar una oportunidad para un nuevo comienzo y cambio.

Así, tratando de construir un nuevo capítulo en su historia y de reconstruir una nueva sociedad libre de conflicto, en los últimos años Colombia ha avanzado en la vinculación de víctimas a la vida civil sin dejar de lado a excombatientes (exguerrilleros y exparamilitares) que han decidido dejar las armas y ser parte de la construcción de la paz, social y económica del país. En este artículo se llevó a cabo una revisión sistemática de la literatura pertinente, a partir de la cual se identificaron y describieron prácticas administrativas que las empresas actualmente implementan en temas de construcción de paz, vinculación de excombatientes y víctimas, a partir de una exploración de artículos relacionados con: 1) construcción de paz en el sector empresarial, 2) reivindicación del posconflicto con las empresas y la sociedad y 3) responsabilidad social empresarial (RSE) como herramienta de mejora en el postconflicto.

\section{Motivación}

Colombia cuenta más de 60 años de conflicto armado interno. Durante este periodo, ha dejado un saldo de más de 261619 víctimas fatales, de las cuales, 46675 fallecieron en combate y 214584 fueron civiles (Observatorio de Memoria y Conflicto, 2018). Es indispensable hacer un recuento tanto de los hechos como de las cifras que hasta el momento ha dejado el conflicto interno en Colombia, con una mirada sobre las víctimas y los desmovilizados que día a día tratan de olvidar su pasado y reconstruir su presente. El desarrollo de esta investigación se enfocó en los aportes realizados por diferentes autores, así como por diferentes entidades: para el 2009, la Asociación Nacional de Empresarios de Colombia (ANDI) 
y Fundación Ideas para la Paz (FIP) realizaron un estudio donde identificaron estrategias para la construcción de paz desde el sector empresarial colombiano.

En ese sentido, instituciones públicas y organizaciones del sector privado han intentado elaborar argumentos económicos apelando al bolsillo, si se quiere, para generar o reforzar la conciencia empresarial en cuanto a los costos en que incurre el sector privado en un contexto de conflicto armado interno (Rettberg, 2008); pero, por otro lado, los investigadores sobre los temas de la paz, a través del comercio, sostienen que las empresas desempeñan un papel importante en la estabilización, reconstrucción y consolidación de la paz después de los conflictos.

Este artículo busca, en particular, tres fines: 1) reunir un marco teórico que permita estudiar las prácticas de rse que promueven la construcción de paz, 2) exponer la construcción de paz y aportes por parte del sector privado a la reconstrucción como país y 3) contribuir a la literatura existente y complementarla en el tema RSE en Colombia en el proceso de paz. Uno de los argumentos que se esbozan para atraer al sector privado como socio de la construcción de paz tiene que ver con su desempeño económico y cómo es afectado por las condiciones de conflicto armado interno (Rettberg, 2007).

De esta manera, es posible llegar a identificar un problema de investigación teniendo en cuenta el vacío literario donde aún no se llegan a identificar investigaciones de carácter detallado para el tema de la vinculación laboral de víctimas y desmovilizados para Colombia; tampoco características de modelos de empleabilidad efectivos e íntegros para esta población. Así, este artículo se encuentra dirigido a empresas, empresarios y académicos colombianos, quienes generan un efecto positivo sobre las prácticas empresariales del posconflicto y aportan a la construcción de paz. 


\section{Metodología}

En esta sección se aborda la metodología utilizada para la investigación, mediante la revisión sistemática de la literatura en las bases de datos bibliográficas para la búsqueda de los artículos, los criterios de clasificación de los artículos, así como las palabras clave.

\subsection{Tipo y enfoque de la investigación}

Esta es una investigación cualitativa con enfoque cuantitativo. "El método de investigación cualitativa no descubre, sino que construye el conocimiento, gracias al comportamiento entre las personas implicadas y toda su conducta observable" (Rodríguez Gómez et al., 1996, p. 6). Por lo tanto, en esta se identifica el papel que desempeña el sector empresarial colombiano y la RSE en la construcción y la creación de la paz.

\subsection{Criterios y datos sobre la búsqueda de la información}

Para esta investigación se analizaron 85 artículos indexados y 15 libros. En el caso de los artículos, se utilizaron bases de datos académicas y científicas como Scopus, Elsevier, Web of Sience y Embase. Así mismo, las palabras para su búsqueda fueron:

- Construcción de paz.

- Responsabilidad social corporativa/empresarial.

- Empresa y construcción de paz.

- Inclusión social.

- Iniciativas de paz.

- Modelos de inclusión. 
Para el caso de los libros, también se utilizaron las bases de datos Ebsco y Scopus. Los criterios para filtrar los artículos y los libros fueron: un periodo publicación entre los años 2009 y 2020, documentos relacionados con el conflicto en Colombia, datos sobre la RSE en Colombia y otra información respecto a otros países que tengan como común denominador la construcción de paz y nuevas oportunidades. Así, pues, toda la presentación de la revisión bibliográfica de este documento se presenta por tema.

\section{Antecedentes y actualidad}

Entre el 2009 y el 2013, en una fase primordialmente de diagnóstico y sensibilización, el trabajo de investigación de la ANDI y de la FIP se enfocó en la sensibilización de empresarios locales sobre papel que podrían desempeñar de cara a la superación del conflicto y la construcción de paz en el país. Esa investigación sustenta que los procesos de administración y funcionamiento de las empresas, su actividad económica y sus inversiones sociales abarcan un compromiso con la paz desde el sector empresarial colombiano. El resultado de tal estudio puede llegar a contribuir a las etapas restantes del proyecto de construir paz a través del sector empresarial en Colombia, como evidencia de que sí es posible reformular la forma en la cual se desarrolla en el país la vinculación laboral de las víctimas y de los desmovilizados.

De igual manera, la RSE se refiere a que a la visión general que se tiene de una empresa deben añadirse otros componentes, por ejemplo: el respeto por los valores éticos, las personas, las comunidades y el medio ambiente; más específicamente, luchar por el respeto de los derechos humanos, ya que es necesario aportar herramientas concretas a las empresas interesadas en vincularse a la construcción de paz, como algunas organizaciones lo están empezando a hacer (Rettberg, 2008).

Asimismo, sugiere que el comportamiento empresarial ético puede ayudar a crear las condiciones necesarias para una paz sostenible, puesto que los estándares de responsabilidad corporativa podrían desempeñar una parte importante en ayudar a formar un comportamiento firme hacia la prevención de los conflictos violentos (Koerber, 2009a). 
Ahora bien, la pregunta de investigación que busca resolver este artículo se centra en ¿qué papel desempeña el sector empresarial colombiano y la RSE en la construcción y la creación de la paz?, ya que se ha logrado evidenciar que en los últimos años la RSE se ha fortalecido, pasando de una tesis filosófica a acciones que forjan impacto.

\section{Revisión de elementos teóricos}

Es necesario identificar los diferentes elementos teóricos que son componentes de esta investigación y también sus aportes, los cuales se complementan entre sí. Una vez superado el conflicto, los países entran en un periodo de posconflicto. Un término usado para referirse globalmente a todos los retos del posconflicto, especialmente la construcción de paz (Ugarriza, 2013).

\subsection{La construcción de paz}

En 1976, Galtung introdujo el concepto de peacebulding o construcción de paz, y de acuerdo con este, al igual que los conflictos llegan a tener una estructura, la paz también tiene una estructura (citado en Mesa, 2008). Está distribución se denomina multinivel o triángulo de la paz, donde Galtung destaca aspectos como el cultural, el social y el político, ya que se debe considerar que la paz debe construirse tanto dentro de las naciones como entre las naciones mismas. En el caso de las Naciones Unidas, en el informe Una agenda para la paz, introducen el enfoque y los conceptos de referencia para el caso de la construcción de paz, un concepto más amplio que se refiere a las acciones para alcanzar una paz estable y duradera y la prevención de conflictos (citado en Mesa, 2008, p. 49).

Igualmente, el Instituto de Estudios sobre Conflicto y Acción Humanitaria (IECAH, 2010), "precisa la construcción de paz (СP) como el conjunto de acciones (en el corto, medio y largo plazo) que permiten a una sociedad prevenir, gestionar y resolver el conflicto por medio de sus propias capacidades, sin tener que recurrir al uso de la violencia" (párr. 1). Por ende, los individuos y la sociedad en su conjunto deben preservar la seguridad y un buen desarrollo sostenible de esta. En un país como Colombia es fundamental fortalecer 
y mejorar los componentes sociales, económicos y empresariales que permitan una construcción de paz sostenible e incluyente, porque es una estrategia, un camino para alcanzar la estabilidad estructural y su correspondiente nivel de seguridad Humana. Trabaja para eliminar las causas estructurales de los conflictos violentos para fortalecer las sociedades y su convivencia pacífica (IECAH, 2010).

Por lo anterior, la construcción de paz es una superación de toda violencia que ejerza el Estado, los grupos privados, los grupos al margen de la ley y otras organizaciones a través de un modelo económico, político o social ético y transformador (Reina-Rozo, 2020). Así mismo, la gestión de un conflicto, sea por parte de un individuo o por una institución, es siempre un proceso dinámico que tiene relación directa con la visión y la forma en que se dan los cambios (Conforti, 2017).

En resumen, la construcción de paz se trata de acometer una auténtica transición, con cambios profundos en dimensiones sociales, empresariales y nacionales (Grasa, 2019). La construcción de la paz es, forzosamente, un enfoque asociativo al conflicto, capaz de hacer frente a lo directo, estructural y a las causas culturales de violencia en su sentido más amplio; por lo tanto, la lucha por la paz se debe hacer de manera integral y equitativa e ir más allá de una discusión teórica.

\subsection{Iniciativas de cambio}

Según el Pacto Global de las Naciones Unidas, las empresas "pueden ayudar a las comunidades locales y a las sociedades en general a lograr sus prioridades de desarrollo y crear oportunidades sostenibles alineadas con sus objetivos estratégicos de negocios" (Gutierres, 2018, p. 11)

En ese orden de ideas, en la revisión sistemática de la literatura sobre modelos de inclusión laboral para la población víctima del conflicto armado y desmovilizados, la información que se puede llegar a encontrar es poca o, en algunos casos, hace hincapié en la descripción de las consecuencias que deja el conflicto armado. Trabajos como el de (Berdal \& Mousavizadeh, 2010)plantean que la construcción de la paz mediante nuevos enfoques de ayuda del sector privado incluyen formas híbridas de actividad estatal y de mercado a la creación de empleos. Por otro lado, el trabajo de Branzei y Abdelnour (2010) 
muestra cómo las empresas generan oportunidad, y así la resiliencia de las empresas produce resultados económicos más favorables.

Otros estudios se encuentran más relacionados con el de esta investigación, como el trabajo de Rettberg (2008), que detalla cómo es la percepción del sector empresarial colombiano del conflicto y presenta alternativas de construcción de paz en Colombia por medio de este sector; así mismo, destacamos el trabajo de Sánchez (2019), el cual se desarrolla en el sector empresarial colombiano (gastronómico) y permite conocer nuevas estrategias empresariales de parte de reincorporados y víctimas que no solo agregan valor, sino que también generan soluciones a la empresa.

Si bien el trabajo de la ANDI y la FIP (2014) resalta que las empresas pueden mantener o incluso exacerbar el conflicto, adaptarse a sus dinámicas o, de manera intencional y proactiva, buscar atenuarlo y robustecer esfuerzos orientados a la construcción de paz, su investigación muestra que generar empleo no necesariamente es una garantía de inclusión, sino que se deben tener componentes que permitan una eficaz inclusión laboral. Es decir, no hay que dejar de lado el proceso de reconciliación y perdón —que es una parte poco desarrollada como eje del desempeño laboral de esta población - por parte de las empresas que generan oportunidades de empleo. Igualmente, el sector empresarial manifiesta que el Gobierno no puede considerar a los empresarios solo como empleadores responsables de ingresos públicos, sino también reconocer su papel específico como constructores de la paz (Cámara de Comercio de Bogotá, Fundación Ideas para la Paz \& Instituto Catalán Internacional para la Paz, 2015).

El aporte del sector empresarial a la construcción de paz, además de llegar a ser una responsabilidad ética y moral, también puede ser vista como una oportunidad de negocio. En la actualidad, en el contexto colombiano, aquellas empresas que logren "capitalizar las oportunidades de negocio que brinda la paz, no solo aportarán al desarrollo socioeconómico justo e inclusivo, sino que además harán una apuesta a largo plazo que traerá importantes retornos" (FIP, 2015, p. 5).

Más aún cuando la paz no suele ser vista como un tema del mundo de los negocios, menos cuando parece convertirse cada vez más en una bandera electoral que no va más allá de las discusiones de quienes están a favor o en contra de las negociaciones (Méndez et al., 2018). 
Por ejemplo, para el 2015 en una encuesta realizada por la Cámara de Comercio de Bogotá (ссв, 2015), el 60\% de los empresarios encuestados afirmó estar de acuerdo con la negociación con las Fuerzas Armadas Revolucionarias de Colombia, ya que indican que es el proceso para alcanzar la paz en el país. De esta forma, se logra identificar el rol y la importancia del sector empresarial colombiano.

En efecto, el papel que desempeñan en la actualidad las empresas puede contribuir de manera positiva a la construcción de paz o, por el contrario, hacer que esta no prospere. ¿Cómo evaluar las contribuciones del sector empresarial? Se distinguen cuatro formas diferentes en que el sector privado puede contribuir a la consolidación de la paz: 1) observando los impactos o contribuciones directas versus las contribuciones indirectas que realiza; 2) teniendo en cuenta las contribuciones explicitas versus las no intencionadas; 3) sopesando las decisiones unilaterales (empresa o sitio único) versus iniciativas conjuntas o empresariales, y 4) analizando contribuciones de paz locales versus las nacionales, regionales o internacionales (Gruener \& Hald, 2015, p. 143).

Es decir, hay tres mecanismos generales por los cuales el sector privado está en capacidad de contribuir a la prevención de conflictos: a través de su negocio y principales actividades, programas de inversión social y participación en diálogos políticos e institucionales (Melin, 2016). De la misma manera, algunos estudios sobre resolución de conflictos y consolidación de la paz han examinado al sector empresarial colombiano y han mostrado el potencial del sector privado, con resultados significativos con programas pioneros en construcción de paz.

Tal como las empresas del sector social y solidario establecieron marcos jurídicos que facilitan y apoyan el emprendimiento solidario en temas como educación, empresas asociativas, cooperativas de servicios, entre otros (Serna-Gómez \& Rodríguez-Barrero, 2015), trabajar con la sociedad es clave, ya que permite la generación de negocios inclusivos, construyendo relaciones productivas gana-gana que no generen dependencia ni responsabilidades exclusivas para el sector empresarial (Cámara de Comercio de Bogotá, Fundación Ideas para la Paz \& Instituto Catalán Internacional para la Paz, 2015).

Las empresas pueden construir paz y, a la vez, obtener mejoras competitivas por hacerlo, lo que ofrece una oportunidad significativa (Fundación Paz y Reconciliación, 2017). Así, 
el aporte del sector empresarial a la construcción de paz, además de llegar a ser una responsabilidad ética y moral, también puede ser vista como una oportunidad de negocio. En la actualidad, en el contexto colombiano aquellas empresas que logren "capitalizar las oportunidades de negocio que brinda la paz, no solo aportarán al desarrollo socioeconómico justo e inclusivo, sino que además harán una apuesta a largo plazo que traerá importantes retornos" (FIP, 2015, p. 5).

Algunas empresas intentan con entusiasmo contribuir a la paz, y un grupo de empresas que se denominan empresarios de paz tienen específicamente esta misión como parte de sus negocios y estrategia (Fort, 2016). Entre estos "empresarios o empresas de paz", encontramos a Terpel, Fundación Corona, Protabaco y Mild Coffee, esta ultima una pyme. A partir de estas experiencias se pudieron medir los impactos de paz en la gestión y la inversión social de cada empresa (FIP, 2017). Las organizaciones empresariales presentan sistemas participativos que pueden ser un modelo para sociedades pacíficas y, por lo tanto, pueden contribuir a la paz más allá del contexto laboral (Spreitzer, 2007).

Durante el proceso de indagación y lectura sobre la contribución del sector empresarial a la creación de paz y el aporte que hacen, también se hallaron empresas que construyen paz y que en la actualidad tienen pautas implementadas que se podrían catalogar como de gran impacto o significativas:

- Entender que aportar a la paz no solamente se logra con la generación de empleo y el pago de impuestos.

- Reconocer que la sostenibilidad de los negocios depende del bienestar de sus entornos y de las personas (clientes, trabajadores y proveedores).

- Invertir en el desarrollo socioeconómico de sus áreas de influencia.

- Ejercer un liderazgo adaptativo e innovador, capaz de identificar oportunidades en situación de incertidumbre.

Por otro lado, es posible que las acciones comerciales fomenten un cambio positivo más amplio, sobre todo para aquellas empresas que priorizan el compromiso con las comunidades 
locales en lugar de responsables políticos (Miklian \& Medina, 2020). En definitiva, diversas iniciativas se han desarrollado e implementado con respecto a la vinculación y creación de empleos para las víctimas y desmovilizados, evidenciando que en Colombia se está intentando un desarrollo socioeconómico incluyente y ecuánime que permite un gran avance del conflicto al posconflicto y la creación de paz.

Ahora bien, a través de una recapitulación de los temas investigados y la relación literaria de cada uno de ellos con la contribución al proceso de inclusión de víctimas y desmovilizados del conflicto armado en Colombia, se llega a la RSE.

\subsection{La responsabilidad social empresarial}

En un intento de agrupar la gran cantidad de definiciones de responsabilidad social empresarial, académicos y empresarios han desarrollado varias formas de agruparlas y categorizarlas, para el caso empresarial se define como: "el compromiso de las empresas de contribuir al desarrollo económico sostenible, trabajando con los empleados, sus familias, la comunidad local y la sociedad en general para mejorar su calidad de vida" (Morros \& Vidal, 2012, p. 35).

En cambio, para Cajiga Calderón (2011), la RSE es un compromiso que tiene toda empresa de cumplir con su finalidad, en la cual se incluyen los aspectos económicos, sociales y ambientales y donde participan los grupos de interés. Para los académicos, la definición se encuentra orientada, en la mayoría de los casos, hacia las pautas y estándares de gestión, que incluye una variedad de mecanismos de cumplimiento (Koerber, 2009). Así, pues, la RSE alude al conjunto de obligaciones, compromisos legales y éticos que se derivan de la actividad de las organizaciones con impacto en las esferas social, laboral, medioambiental y de los derechos humanos (Avendaño, 2013). Por lo que la RSE se entiende como el reconocimiento e integración en sus operaciones que da lugar a prácticas empresariales que satisfagan dichas preocupaciones y configuren sus relaciones con la sociedad en general.

En relación con la RSE en contextos de conflicto armado, la empresa debe estar al tanto del contexto de conflicto en el que opera y sus actores, de modo que adapte su negocio y las relaciones con sus stakebolders desde una perspectiva de "sensibilidad al conflicto" (Prandi \& Lozano, 2011). Ahora bien, el modelo stakebolder favorece el involucramiento 
organizacional en la RSE teniendo en cuenta tres factores clave: 1) la obligación social y legal, 2) las metas de responsabilidad social de la empresa/beneficio social y 3) el anticiparse o transformar problemas sociales (Barrios Hernández, 2016).

El posconflicto resulta ser un espacio adecuado para que las organizaciones reconsideren su RSE y se preparen para vincular a los excombatientes en el ámbito social y laboral (Téllez, 2018). Según los datos de la primera encuesta realizada por el Centro Nacional de Memoria Histórica a las víctimas en el 2013, solo el $2.8 \%$ tiene un empleo formal que garantice el cumplimiento al artículo 53 de la Constitución Política de Colombia, el cual normativiza "la igualdad de oportunidades para los trabajadores; remuneración mínima vital y móvil, proporcional a la cantidad y calidad de trabajo; estabilidad en el empleo". Otra de las cifras que revela la encuesta es el porcentaje de víctimas que labora en la informalidad, siendo un $41.2 \%$ de esta población.

Además, solo un 19\% de la ocupación laboral en el sector empresarial está dispuesto a emplear a un desmovilizado. Cabe resaltar que sectores como construcción, agropecuario, industrial y de servicios tienen una disposición del 100\% de vincular dentro de su nómina a personas desmovilizadas; mientras que los sectores financiero y comercial evidencian una negativa del 100\% en contratar desmovilizados (Roldán Castellanos, 2013). Para Barrios Hernández (2016), la RSE ética es el medio por el cual las organizaciones desarrollan las acciones que generarán impacto social y que garantizan la vinculación de los actores al mercado laboral.

Hoy en día se reconoce la necesidad de la participación de las empresas en los procesos de consolidación de la paz, y todavía es un tema pendiente en Colombia (LópezSantamaría \& Grueso Hinestroza, 2019). Con motivo de lo anterior, la Organización para la Cooperación y el Desarrollo Económicos (2013) generó unas directrices tanto para los gobiernos como para las empresas donde el papel de las empresas es respetar los derechos humanos de las personas que pertenecen a categorías específicas o poblaciones que merecen una atención particular.

De esta manera, la RSE se constituye en un elemento determinante para lograr la igualdad de oportunidades en el empleo. Pretende, además, analizar el concepto rse desde la normatividad, con el fin de posicionarla más allá de una actividad altruista que deberían implementar las organizaciones (Grueso Hinestroza, 2009)aun- que en condiciones de 
inequidad. Uno de los modelos de responsabilidad social empresarial (RSE. Por otra parte, todas las empresas tienen la posibilidad de implementar estrategias y acciones para la paz, sin importar su tamaño, ubicación, nacionalidad y el sector al que pertenecen. La RSE es dinámica, porque cambia con el tiempo del mismo modo que lo hace la sociedad (incluyendo a los propios empresarios y accionistas) y con las nuevas necesidades y exigencias de esa sociedad (Peribáñez, 2017).

Entre tanto, la experiencia colombiana de inclusión de poblaciones víctimas y desmovilizados del conflicto a modelos de trabajo que permitan un avance más efectivo en la reparación social ha sido la apuesta del sector empresarial por medio de la RSE (LizcanoPrada \& Lombana, 2018); pero cabe destacar que en la actualidad solamente algunas empresas han realizado aproximaciones a partir de iniciativas, logrando una inclusión integral y no parcial.

Es el caso del Grupo Éxito, que desarrolla un programa de inclusión laboral para poblaciones vulnerables y brinda oportunidades de formación para el trabajo e inserción laboral a personas que la sociedad margina por consecuencia de la violencia urbana o rural (Prandi \& Lozano, 2011). Así mismo, un modelo empresarial en el que están articuladas iniciativas de perdón y reconciliación es el restaurante El Cielo, que ha logrado capacitar en el arte de la cocina a alrededor de 600 personas entre víctimas y desmovilizados del conflicto en nuestro país (Sierra Rincón \& Álvarez, 2019). No obstante, un gran porcentaje del tejido empresarial en Colombia son las pymes, donde existe una falsa creencia de que la RSE es exclusiva de las grandes empresas. Ello es una de las principales barreras en el momento de acercarse a la responsabilidad empresarial como una nueva forma de hacer negocios (Sepúlveda Romero et al., 2016).

Por otra parte, tradicionalmente se señalan como beneficios de la RSE la mejora de la reputación empresarial, el fortalecimiento de la lealtad de los consumidores, la mejora en la calidad y la productividad, el aumento de la rentabilidad, entre otros tantos (Gómez Nieto \& Martínez Domínguez, 2016). "La RSE es un concepto multidimensional y ponen de manifiesto que la RSE desempeña un rol como variable antecedente de la imagen y la reputación empresarial" (Alvarado, A, 2008, p. 42). 
A partir de ello se entiende como inclusión integral aquella que en su componente de desarrollo maneja cuatro grandes dimensiones de intervención (desarrollo socioeconómico inclusivo, capital humano, reconciliación y convivencia y sostenibilidad ambiental). De tal manera, con el propósito:

[De que] las empresas puedan tener un impacto significativo en la construcción de paz, es necesario implementar acciones que alineen las estrategias empresariales y del sector con apuestas productivas municipales y regionales, mediante la incidencia en políticas y programas público-privados en aras de un desarrollo regional inclusivo y equitativo. (FIP, 2015, p. 24)

Para concluir, en la actualidad, las empresas llevan a cabo muchas innovaciones y cambios para contribuir a la reconciliación del país; pero también tienen la responsabilidad de posicionarse y convertirse en una empresa sustentable, ya que a partir de esas iniciativas de perdón y construcción de paz se evidencia que el sistema económico devuelve a la sociedad en gran medida el apoyo y los beneficios recibidos en el mercado.

\section{Discusión}

\subsection{Construcción de paz e iniciativas de cambio}

En los últimos años algunas empresas han tenido la iniciativa de que en su planta de personal sean contratadas personas que de alguna manera pertenecieron al conflicto armado en Colombia; pero en el proceso de vinculación y en la etapa de empleabilidad no manejan programas de sensibilización en el interior de la empresa, tampoco en las áreas en las que la víctima o el desmovilizado desarrollan su trabajo.

En relación con el sector privado, al menos por su estabilidad, no se puede abandonar cierto grado de reacción y responsabilidad, especialmente necesarias en situaciones de conflicto y posconflicto, en las que se requieren disposiciones para crear nuevas herramientas que se ajusten a este tipo de situaciones (Prandi \& Lozano, 2011, p. 163). Así mismo, 
las empresas internacionales aportan experiencia y finanzas, al igual que un compromiso duradero para la construcción de paz (Bray, 2005).

En esta sociedad, tales iniciativas — que hasta el momento solo nacen del sector empresarial— no son correspondidas por la sociedad civil, lo que genera más brechas en el proceso de inclusión para este tipo de población. Por ello, es necesario que el Estado construya una serie de políticas públicas que estimulen la inclusión de los desmovilizados al mercado laboral, de forma subordinada o independiente (Roldán Castellanos, 2013). Colombia ha sido parte de la conformación de negocios que aportan a la construcción de paz, y estrictamente hablando, a la mitigación de las causas o efectos del conflicto armado tanto en zonas geográficas concretas como en grupos de población específica (Grasa, 2019).

Durante el proceso de investigación, se logró evidenciar que los diferentes actores del conflicto (víctimas y victimarios) muestran su interés por respaldar la construcción de paz en el país; sin embargo, el sector empresarial debe realizar un proceso de reintegración que abarque todos los aspectos que influyen en el óptimo desarrollo de los reinsertados y de las víctimas.

Las iniciativas de cambio que a lo largo de esta investigación se lograron hallar para el caso colombiano se destacan por cuatro características que generan construcción de paz:

- Integraciones sociales entre víctimas y desmovilizados para la generación de oportunidades productivas que fomenten la empleabilidad y el ingreso al mercado laboral.

- Desarrollo de clústeres para la producción y comercialización agroindustrial en zonas afectadas por el conflicto. Esta iniciativa cuenta con el apoyo de la empresa Bavaria y el Departamento de Prosperidad Social.

- Fortalecimiento de la economía de sectores afectados por el conflicto armado a través de la vinculación de empresas a estos territorios y desarrollo de proyectos que generen beneficios sociales. 
- Creación de cadenas de valor de empresas a través de capacitaciones y fomento de la empleabilidad.

\subsection{Construcción de paz y responsabilidad social empresarial}

El sector empresarial, por medio de la RSE en los últimos años y más específicamente a partir de la firma del Acuerdo de Paz en Colombia, ha sobresalido por su voluntad de impulsar programas y asociaciones de práctica que trabajen por la construcción de paz en el país. En otras palabras, la empresa se ha reivindicado por ser un actor clave en la gobernanza de asuntos públicos y en la prevención y mitigación de problemas sociales en las áreas donde su presencia es influyente (Grasa, 2019, p. 93).

La RSE tomada en serio ampliaría el alcance del mecanismo político para toda actividad humana. No difiere en filosofía de la mayoría doctrina explícitamente colectiva (Friedman, 2007). Ello llevaría a la construcción de paz, al definirse visiblemente un propósito social, porque saberlo integrar a los procesos de la empresa, implica el fomento de una cultura de paz en la cual las prácticas de la RSE llegan a tener gran impacto en el ámbito socioeconómico de un país.

Una de las conclusiones en cuanto a la solución que brinda la RSE en la construcción de paz se logra evidenciar en que algunas empresas utilizan esta herramienta para brindar inclusión tanto a víctimas como a desmovilizados, pero no llegan a medir los resultados y sus proyectos, en algunos casos. Por otro lado, otras empresas utilizan estos programas para mejorar la percepción reputacional de la marca o empresa; pero sin un verdadero propósito social (Banks, 2016), por lo que los resultados de sus programas o iniciativas no logran demostrar su contribución a la construcción de paz.

\subsection{Iniciativas de cambio y responsabilidad social empresarial}

Las iniciativas de cambio se trasforman según el contexto y el propósito de la compañía. Todas ellas, en general, tienen por objetivo aumentar el valor de la empresa para que esta sea más competitiva, mejorar la satisfacción de los trabajadores, generar responsabilidad e independencia en ellos, además generar oportunidades de inserción laboral, que para este caso son las víctimas y los desmovilizados. La RSE debe iniciar adentro de las compañías, 
de manera transparente, actuaciones éticas, con procesos que minimicen impactos negativos en el ambiente, en las personas y en la sociedad; pero que también optimicen sus relaciones con los actores sociales a quienes sus operaciones pueden impactar, negativa o positivamente (Hernández, 2016).

La relación entre la RSE y las iniciativas de cambio implica el compromiso de darles solución a los efectos e implicaciones que ha dejado el conflicto armado en nuestro país. Las empresas, por medio de la RSE, son socialmente responsables de orientar la satisfacción de las necesidades y expectativas de la sociedad y de quienes se benefician de su actividad comercial, así como el cuidado y preservación del entorno; por otro lado, hay que pensar la RSE como un proceso interactivo que permite conocer y aprender del otro, crecer y desarrollarse. En definitiva, coconstruir no solo para la empresa, sino también para la sociedad (Conforti, 2018).

En definitiva, las iniciativas de cambio agregan valor a la empresa en su conjunto, cambian la forma de pensar y actuar de las empresas, potencian su imagen y las llevan hacia la eficiencia competitiva, es decir, las transforma en competidores éticos y eficientes, dejando de lado el mito de que utilizar la RsE solo hace parte del cumplimiento normativo para fortalecer una marca y disminuir impuestos.

\section{Conclusiones}

Por otra parte, cuando se trata de inclusión social y laboral, no son solamente las grandes empresas los motores del cambio; son las pymes las verdaderas inductoras del nuevo modelo de sociedad (Serna-Gómez \& Rodríguez-Barrero, 2015).

En cuanto a esta investigación, busca aportar y demostrar que sí es posible un desarrollo social inclusivo desde el sector empresarial. En la actualidad, estos logros se evidencian cuando las empresas emplean a personas afectadas por el conflicto, porque con ello garantizan un trabajo digno y apoyan los negocios inclusivos y proyectos locales que ejercen tanto víctimas como desmovilizados, ya que la paz es más comúnmente una 
condición o una situación para unificar un país y fortalecer el papel empresarial (Forrer \& Fort, 2016). Esta última alternativa es una nueva propuesta respecto a cómo implementar los principios del valor compartido. La economía compartida promete una distribución más amplia de beneficios entre los interesados, lo que puede influir en el atractivo de los inversores (De Lange, 2020).

Investigaciones futuras deben considerar otros elementos que influyen en la decisión de apoyar prácticas de posconflicto, como la percepción que se tiene en general del proceso, el impacto que esto genera en las organizaciones y las garantías de participación. Dentro de los resultados de esta investigación también queda en el aire la postura política del empresariado colombiano frente a los roles que debe asumir en el posconflicto, especialmente empresarios de las pymes, quienes aun con la evidencia bibliográfica descrita y según la gran encuesta a las microempresas solamente un $2.1 \%$ busca tener prácticas de RSE (ANIF, 2018).

Así mismo, el papel que desempeñan las universidades y la entidades académicas en la promoción de la paz, en materia académica no se deben limitar a las referencia teorías académicas, ya que este tema abarca muchas áreas en general (Katsos \& Fort, 2016). Por otro lado, Porter y Kremer "Indican que las necesidades de la sociedad, y no solo las necesidades económicas convencionales, son las que definen los mercados" (2011, p. 5), dado que crear una sociedad exitosa también depende del fortalecimiento del sistema educativo y de la contribución que genere la academia.

Para finalizar, otro de los temas que se quisiera articular es que el Estado se refirió a la reincorporación como uno de los mayores desafíos y que estaba impulsando todas las acciones necesarias, pero actualmente las empresas dicen no recibir el apoyo del Estado (Fajardo España \& Murcia Báquiro, 2018). Los críticos de la paz liberal han acusado que las intervenciones de apoyo a la paz se ejercieron para el Estado y no para la empresa (Brett \& Malagón, 2013). Una de las conclusiones a las que se ha llegado al estudiar y en el análisis de la revisión sistemática de la literatura es que las iniciativas de cambio, la RSE y la construcción de paz establecen una hoja de ruta en proceso de paz para nuestro país, pero que el verdadero compromiso nace de cada uno de nosotros. 


\section{Referencias}

Asociación Nacional de Empresarios de Colombia (ANDI), Fundación Ideas para la Paz (FIP). (2014). ¿Cómo construir paz desde el sector empresarial en Colombia?

Alvarado, A. (2008). Dimensionalidad de la responsabilidad social empresarial. Estudios Gerenciales, 24(108), 37-59.

Asociación Nacional de Instituciones Financieras (ANIF). (2018). Gran encuesta a las microempresas: Informe de resultados. https://www.anif.com.co/sites/default/files/publicaciones/ gem18_.pdf

Avendaño, W. (2013). Responsabilidad social (Rs) y responsabilidad social corporativa (RCs): Una nueva perspectiva para las empresas. Revista Lasallista de Investigación, 10(1), 152-163.

Barrios Hernández, R. (2016). Responsabilidad social empresarial: El papel de las organizaciones en el postconflicto colombiano. Revista de Estudiantes de Administración de Empresas, 9(1).

Banks, H. (2016). The business of peace: Coca-Cola's contribution to stability, growth, and optimism. Business Horizons, 59(5), 455-461. https://doi.org/10.1016/j.bushor.2016.03.018

Bray, J. (2005). International companies and post-conflict reconstruction. University of Arizona Libraries. https://doi.org/10.2458/azu_acku_pamphlet_hf3770_6_z5_b73_2005

Berdal, M., \& Mousavizadeh, N. (2010). Investing for peace: The private sector and the challenges of peacebuilding. Survival, 2(52), 37-58.

Branzei, O., \& Abdelnour, S. (2010). Another day, another dollar: Enterprise resilience under terrorism in developing countries. Journal of International Business Studies, 41(5), 804-825.

Brett, R., \& Malagón, L. (2013). Overcoming the original sin of the "original condition": How reparations may contribute to emancipatory peacebuilding. Human Rights Review, 14(3), 257-271. https://doi.org/10.1007/s12142-013-0271-5

Cajiga Calderón, J. F. (2011). El concepto de responsabilidad social empresarial. Centro Mexicano para la Filantropía (Cemefi). http://www.cemefi.org/esr/images/stories/pdf/ esr/concepto_esr.pdf

Cámara de Comercio de Bogotá (ССв). (2015). Encuesta empresarial sobre construcción de paz.

Cámara de Comercio de Bogotá, Fundación Ideas para la Paz, \& Instituto Catalán Internacional para la Paz. (2015). Perspectivas y aportes empresariales para la construcción de paz. http://hdl.handle.net/11520/8482

Conforti, F. (2017). Construcción de paz: Diseño de intervención en conflictos. Dykinson. 
Conforti, F. (2018). Integridad: El aporte de organizaciones y empresas a la construcción de paz. Dykinson. https://doi.org/10.2307/j.ctt22p7gxh

De Lange, D. (2020). Preferencias de los inversores entre la economía colaborativa y las empresas establecidas. Ted Rogers School of Management.

Fajardo España, M., \& Murcia Báquiro, P. A. (2018). Inclusión laboral de desmovilizados en un estudio sobre los tipos empleos de las últimas décadas en Colombia y los organismos internacionales oNGS [tesis de pregrado]. Universidad Piloto de Colombia. http://repository.unipiloto.edu.co/handle/20.500.12277/4906

Forrer, J. J., \& Fort, T. L. (2016). The PaCo index. Business Horizons, 59(5), 533-538. https:// doi.org/10.1016/j.bushor.2016.03.017

Fort, T. L. (2016). The business of pace. 59(5).

Fundación Ideas para la Paz (FIP). (2015). ¿Cómo construir paz desde las empresas? Guía práctica. http://cdn.ideaspaz.org/media/website/document/590fb882ac88a.pdf

Fundación Ideas para la Paz (FIP). (2017, 4 de mayo). Abora los empresarios cuentan con una herramienta para medir su aporte a la paz. http://www.ideaspaz.org/publications/ posts/1509

Fundación Paz y Reconciliación. (2017). Guía para construir la paz desde el sector empresarial. https://empresasypaz.pares.com.co/index.html\#slide2

Friedman, M. (2007). The social responsibility of business is to increase its profits. En W. C. Zimmerli, M. Holzinger, \& K. Richter (Eds.), Corporate ethics and corporate governance (pp. 173-178). Springer. https://doi.org/10.1007/978-3-540-70818-6_14

Gómez Nieto, B., \& Martínez Domínguez, R. (2016). Los valores éticos en la responsabilidad social corporativa. Anagramas. Rumbos y Sentidos de la Comunicación, 14(28), 33-50. http://hdl.handle.net/11407/2358

Grasa, R. (2019). Construcción de paz y valor compartido. Icono.

Gruener, S., \& Hald, M. (2015). Inclusive peacebuilding recognised but not realised. Dag Hammarskjöld Foundation.

Grueso Hinestroza, M. P. (2009). Responsabilidad social empresarial e igualdad de oportunidades en el empleo: ¿Altruismo o legalidad? Universidad E Empresa, 11(17), 244-251. https://revistas.urosario.edu.co/index.php/empresa/article/view/1091

Instituto de Estudios sobre Conflictos y Acción Humanitaria (IECAH). (2010, 20 de noviembre). Construcción de la paz. https://iecah.org/index.php/investigacioncp

Katsos, J., \& Fort, T. (2016). Liderazgo en la promoción de la paz: Entrevistas con los homenajeados de Business for Peace 2015. Horizontes de Negocios, 59(5), 463-470. 
Koerber, C. P. (2009). Corporate responsibility standards: Current implications and future possibilities for peace through commerce. Journal of Business Ethics, 89(S4), 461-480. https://doi.org/10.1007/s10551-010-0397-1

Lizcano-Prada, J., \& Lombana, J. (2018). Responsabilidad social corporativa (RSC): Reconsiderando conceptos y enfoques. Civilizar Ciencias Sociales y Humanas, 18(34), 119-134.http://doctrina.vlex.com.co/vid/responsabilidad-social-corporativa-rsc-745911409

López-Santamaría, M., \& Grueso Hinestroza, M. P. (2019). Business, managers and peacebuilding in Colombia: Findings and challenges. Revista de Ciencias Sociales, 25(1), 146-155.

Melin, M. M. (2016). Business, peace, and world politics: The role of third parties in conflict resolution. Business Horizons, 59(5), 493-501. https://doi.org/10.1016/j.bushor.2016.03.013

Méndez, M. L., Noordam, K. M., \& Sánchez Barrera, J. F. (2018, 29 de enero). Diez preguntas para saber si su empresa le aporta a la paz. Fundación Ideas para la Paz. http://www. ideaspaz.org/publications/posts/1640

Mesa, M. (2008). La prevención de conflictos y la construcción de la paz en el seno de Naciones Unidas: De las palabras a la acción. Centro de Educación e Investigación para la Paz. https://ceipaz.org/wp-content/uploads/2020/05/02.-manuela-mesa.pdf

Miklian, J., \& Medina, J. (2020). Theorizing business and local peacebuilding through the "Footprints of Peace" coffee project in rural Colombia. Business \& Society, 59(4), 676-715. https://doi.org/10.1177/0007650317749441

Morros, J., \& Vidal, I. (2012). Responsabilidad social corporativa. FC Editorial.

Observatorio de Memoria y Conflicto. (2018). Balance del conflicto armado [Infografía]. http://centrodememoriahistorica.gov.co/observatorio/infografias/

Organización para la Cooperación y el Desarrollo Económicos. (2013). Líneas directrices de la OCDE para empresas multinacionales. https://doi.org/10.1787/9789264202436-es

Peribáñez, B. (2017). Responsabilidad social corporativa: El papel de las Naciones Unidas y otras organizaciones internacionales en la promoción de la RSC. Dykinson.

Prandi, M., \& Lozano, J. M. (2011). La RSE en contextos de conflicto y postconflicto: De la gestión del riesgo a la creación de valor. Instituto de Innovación Social.

Porter, M. E., \& Kremer, M. R. (2011, enero-febrero). La creación de valor compartido. Harvard Business Review. América Latina. 1-18. http://www.iarse.org/uploads/Shared\%20Value\%20 in\%20Spanish.pdf

Reina-Rozo, J. D. (2020). Ingeniería para la construcción de paz: Una reflexión preliminar para procesos tecnocientíficos de resiliencia territorial. Revista Opera (27), 141-162. https:// doi.org/10.18601/16578651.n27.07 
Rettberg, A. (2008). Explorando el dividendo de la paz: Percepción de los impactos del conflicto armado en el sector privado colombiano. Resultados de una encuesta nacional. Ediciones Uniandes-ceso. https://appsciso.uniandes.edu.co/sip/data/pdf/Explorando_el_dividendo_de_la_paz.pdf

Rodríguez Gómez, G., Gil Flores, J., \& García Jiménez, E. (1996). Metodología de la investigación cualitativa. Aljibe.

Roldán Castellanos, L. (2013). La inclusión laboral de los desmovilizados del conflicto en Colombia: Auténtico mecanismo emancipador de la violencia en Colombia. Universitas Estudiantes (10), 103-119.

Sánchez, M. (2019). Sistematización restaurante El Cielo. Bogotá: Pontificia Universidad Javeriana.

Salas-Arbeláez, L., García- Solarte, M., \& Azuero-Rodríguez, A. R. (2020). Efecto de la responsabilidad social empresarial sobre el posconflicto colombiano: El caso de las pymes. Estudios Gerenciales, 36(154), 80-90. https://doi.org/10.18046/j.estger.2020.154.3547

Sepúlveda Romero, M. E., Moreno Martínez, E. P., Tovar Mesa, J. E., Franco Villalba, J. A., \& Villarraga Tole, C. L. (2016). Responsabilidad social de las Pymes en el marco del posconflicto, como soporte para la inclusión laboral de la población reinsertada. Comunicación, Cultura y Política, 6(1), 45. https://doi.org/10.21158/21451494.n1.2015.1335

Serna-Gómez, H., \& Rodríguez-Barrero, M. S. (2015). El sector solidario como alternativa para el desarrollo social e inclusivo en el posconflicto colombiano. Revista Cooperativismo y Desarrollo, 23(107), 37-48. https://doi.org/10.16925/co.v23i107.1250

Sierra Rincón, S., \& Álvarez, S. V. (2019). Modelo empresarial de inclusión laboral para víctimas y desmovilizados del conflicto armado [tesis de maestría]. Universidad del Rosario, Colombia. https://repository.urosario.edu.co/handle/10336/20308

Spreitzer, G. (2007). Giving peace a chance: Organizational leadership, empowerment, and peacey. Journal of Organizational Behavior, 28(8), 1077-1095. https://doi.org/10.1002/ job.487

Téllez, C. A. (2018). Empresas, responsabilidad social y posconflicto en Colombia. Ascolfa.

United Nations. (2018). Pacto Mundial de las Naciones Unidas: Una llamada a la acción para empresas sostenibles. https://www.pactomundial.org/wp-content/uploads/2018/02/FlyerNew-Strategy-GC-2018_20180126.pdf

Ugarriza, J. E. (2013). La dimensión política del postconflicto: Discusiones conceptuales y avances empíricos. Colombia Internacional (77), 141-176. http://dx.doi.org/10.7440/ colombiaint77.2013.06 